\title{
A Proteome Reference Map of the Causative Agent of Melioidosis Burkholderia pseudomallei
}

\author{
Patompon Wongtrakoongate, ${ }^{1}$ Sittiruk Roytrakul, ${ }^{2}$ Sukkid Yasothornsrikul, ${ }^{3}$ \\ and Sumalee Tungpradabkul ${ }^{1}$ \\ ${ }^{1}$ Department of Biochemistry, Faculty of Science, Mahidol University, Bangkok 10400, Thailand \\ ${ }^{2}$ National Center for Genetic Engineering and Biotechnology, National Science and Technology Development Agency, \\ Pathumthani 12120, Thailand \\ ${ }^{3}$ Department of Biochemistry, Faculty of Medical Science, Naresuan University, Phitsanulok 65000, Thailand
}

Correspondence should be addressed to Patompon Wongtrakoongate, neocmir@hotmail.com and Sumalee Tungpradabkul, pexcotung@gmail.com

Received 15 May 2011; Revised 6 July 2011; Accepted 23 July 2011

Academic Editor: Don Mark Estes

Copyright (C 2011 Patompon Wongtrakoongate et al. This is an open access article distributed under the Creative Commons Attribution License, which permits unrestricted use, distribution, and reproduction in any medium, provided the original work is properly cited.

\begin{abstract}
Burkholderia pseudomallei is the etiologic agent of melioidosis. Using 2DE and MALDI-TOF MS, we report here a proteome reference map constructed from early stationary phase, a bacterial adaptation process. We identified 282 protein spots representing 220 ORFs; many of them have been implicated in bacterial pathogenesis. Up to $20 \%$ of identified ORFs belong to posttranslational modification and stress responses. The proteome reference map will support future analysis of the bacterial gene and environmental regulation and facilitate comparative proteomics with its sibling species.
\end{abstract}

\section{Introduction}

Burkholderia pseudomallei is a pathogenic bacterium causing melioidosis disease, which is endemic predominantly in south Eastern Thailand and Northern Australia. It has also been recognized as a B-type biological warfare agent possessing an ability for bioterrorism [1]. Its sibling species $B$. malle $i$ is the parasitic bacterial pathogen of Glanders disease and was recognized for its destructive role during the World War I [2]. In contrast, another closely related species previously characterized as a $B$. pseudomallei strain with an ability of arabinose-positive assimilation, that is, $B$. thailandensis, is unable to invoke pathogenesis in human. However, these bacterial species harbor genomic DNA with high similarity in nucleotide as well as in amino acid sequences, suggesting that gain and/or loss of genes during evolution is a reason for differences in their behavior and pathogenicity [3-6]. For example, an arabinose catabolic operon belonging to $B$. thailandensis, which is absent in both $B$. pseudomallei and B. mallei genomes, has a suppressive role in $B$. pseudomallei virulence identified by the operon overexpression analysis [5]. In addition, using a comparative proteomics of proteins expressed at prolonged stationary phase, we could identify protein-encoding genes that are lost in B. thailandensis genome compared with that of B. pseudomallei [7]. Therefore, an establishment of a 2DE dataset proteome reference map from one of these three species will be a valuable resource for comparing protein expression profile in order to study their pathogenesis differentially caused by $B$. pseudomalle $i$ and $B$. malle $i$ comparing with $B$. thailandensis. To this end, given the fact that $B$. pseudomalle $i$ has the most biomedical impact between these three bacterial species, the proteome reference map constructed from $B$. pseudomallei will eventually lead to the most effective effort, not only for interspecies comparative proteomic analysis [7] but also for studying gene and environmental regulation in this bacterium [8-10].

We previously reported an initiation of a partial proteome reference map of $B$. pseudomallei grown at prolonged stationary phase to apply for a proteomic comparison between this virulent species and the nonvirulent species B. thailandensis [7]. However, only 88 protein spots were 
identified at this growth phase limiting the application on further analysis of other growth conditions due to the spatial and temporal control of protein expression in this bacterium. To further expand the characterization of protein expression profile in B. pseudomallei, we performed an extended proteome reference map of this bacterium grown at early stationary phase. The early stationary stage of growth has been shown to be relevant to an adaptation phase in bacteria reflected by the gene regulatory pattern of the adaptive stress response RpoS [11]. We have successfully identified 282 protein spots representing 220 ORFs. This proteome reference map will be an invaluable data for further analysis of pathogenesis and virulence factors of B. pseudomalle $i$ and its sibling species.

\section{Materials and Methods}

2.1. Bacterial Culture and Protein Extraction. The clinical isolated B. pseudomallei strain 844 [12] was grown in $100 \mathrm{~mL}$ Luria Bertani (LB) medium at $37^{\circ} \mathrm{C}$ and $200 \mathrm{rpm}$ [9]. Bacterial cells were pelleted using the culture at the 12th $h$ of growth $\left(\mathrm{OD}_{600}\right.$ of about 9.0) corresponding to the early stationary phase by centrifugation at $10,000 \times \mathrm{g}$ and $4^{\circ} \mathrm{C}$. Protein was extracted by resuspending the cell pellet in $500 \mu \mathrm{L}$ lysis buffer ( $8 \mathrm{M}$ urea, $4 \% \mathrm{w} / \mathrm{v}$ CHAPS, $2 \mathrm{mM}$ TBP, $1 \% \mathrm{v} / \mathrm{v}$ IPG buffer $\mathrm{pH}$ 4-7 (Amersham Biosciences, Uppsala, Sweden) and $1 \% \mathrm{v} / \mathrm{v}$ protease inhibitor cocktail set II (Calbiochem, La Jolla, CA)). Cell lysis was performed by sonication on ice. Cell debris was then removed by centrifugation at $13,000 \times \mathrm{g}$ for $30 \mathrm{~min}$ at $20^{\circ} \mathrm{C}$. Protein concentrations were determined using $R C D C$ protein assay kit (BioRad, Hercules, Calif, USA) as previously described [7].

2.2. Two-Dimensional Gel Electrophoresis. 2DE was performed as previously described [9]. 2DE gels were fixed in fixing solution $(50 \% \mathrm{v} / \mathrm{v}$ ethanol and $2 \% \mathrm{v} / \mathrm{v}$ phosphoric acid) for $1 \mathrm{~h}$. The gels were washed by soaking in distilled water for $10 \mathrm{~min}$. The proteins were then stained in the staining solution $(20 \% \mathrm{v} / \mathrm{v}$ methanol, $10 \% \mathrm{v} / \mathrm{v}$ phosphoric acid, $10 \% \mathrm{w} / \mathrm{v}$ ammonium sulfate, and 0.1\% CBBG-250) for an overnight staining. The $2 \mathrm{DE}$ gels were scanned with the ImageScanner (Amersham Biosciences). Image analysis was performed using the PDQuest software version 7.1.1 (BioRad). A master gel used for spot-matching process was created. The master gel was then used to match corresponding protein spots between 2DE gels from three different cultures. Approximately 700 protein spots were detected using the software. Approximately 300 protein spots were picked manually, and they were subjected to tryptic ingel digestion.

2.3. Tryptic In-Gel Digestion. Protein spots were excised from a 2DE gel and transferred into $1.5 \mathrm{~mL}$ microcentrifuge tubes. The in-gel digestion was carried out in 96-well microtiter plates by the Ettan Spot Handling workstation (Amersham Biosciences). The gel pieces were washed in $200 \mu \mathrm{L}$ washing solution $(50 \% \mathrm{v} / \mathrm{v}$ methanol and $5 \%$ acetic acid) for $2 \mathrm{~h}$ at room temperature and were dehydrated by the addition of
$200 \mu \mathrm{L}$ acetonitrile. An incubation was performed for $5 \mathrm{~min}$, and the gels were completely dried in a vacuum centrifuge. Protein disulfide bonds were reduced by adding $30 \mu \mathrm{L}$ of $10 \mathrm{mM}$ DTT and incubating for $1 \mathrm{~h}$ at $56^{\circ} \mathrm{C}$. They were then alkylated by adding $30 \mu \mathrm{L} 100 \mathrm{mM}$ iodoacetamide and incubating for $1 \mathrm{~h}$ at room temperature. The gel pieces were dehydrated in $200 \mu \mathrm{L}$ acetonitrile for $5 \mathrm{~min}$ to exchange the buffer. Two hundred $\mu \mathrm{L}$ of $100 \mathrm{mM}$ ammonium bicarbonate was added to rehydrate the gel pieces. The gel pieces were dehydrated again in $200 \mu \mathrm{L}$ acetonitrile for $5 \mathrm{~min}$ and completely dried in a vacuum centrifuge for $3 \mathrm{~min}$. The tryptic digestion was carried out for overnight at $37^{\circ} \mathrm{C}$ with $30 \mu \mathrm{L}$ of $20 \mathrm{ng} / \mu \mathrm{L}$ ice-cold, sequencing-grade trypsin (Promega, Madison, Wis, USA). Peptides produced after digestion were collected by extraction with $30 \mu \mathrm{L}$ of $20 \mathrm{mM}$ ammonium bicarbonate and $30 \mu \mathrm{L}$ of $50 \%$ acetonitrile with $5 \%$ formic acid. The peptide extracts were then collected into 96-well microtiter plates for further analysis.

2.4. Protein Identification. For protein identification using the matrix-assisted laser desorption ionization time-offlight mass spectrometry (MALDI-TOF MS), peptide mass fingerprinting (PMF) was analyzed using the Reflex IV MALDI-TOF mass spectrometer (Bruker Daltonik, Bremen, Germany). The matrix solution (10\% w/v 2,5-dihydroxy benzoic acid (Sigma, St. Louis, Mo, USA) in 50\% v/v acetonitrile with $0.05 \mathrm{v} / \mathrm{v}$ trifluoroacetic acid (TFA)) was prepared prior to use. Peptide extracts were solubilized in 96-well microtiter plates by adding $2 \mu \mathrm{L}$ of $0.1 \%$ TFA. One $\mu \mathrm{L}$ of the solubilized peptides were mixed with an equal volume of the matrix solution on a 384-well AnchorChip target plate (Bruker Daltonik, Bremen, Germany). The spots were dried at room temperature until crystal occurred, and the target plate was then subjected to the PMF analysis. The mixture was irradiated with a $337 \mathrm{~nm} \mathrm{~N} 2$ laser and accelerated with $20 \mathrm{kV}$ accelerating voltage in a two-stage gridless pulsed ion extraction source. The mass spectrometer was operated in the positive reflectron mode. The covered peptide masses ranged between 800 to 3,500 Dalton. The resulting PMF of each protein spot was visualized by the flexAnalysis software version 2.2 (Bruker Daltonik). Protein identification from the tryptic fragment sizes was performed by using PMF searching against the bacterial subgroup of "other proteobacteria" using the entire NCBI'sNR protein database from the MASCOT search engine (http://www.matrixscience.com/). We set the assumption as monoisotopic peptides, a fixed modification at cysteine residues and a variable oxidation at methionine residues. Up to one missed tryptic cleavage was also allowed [7].

\section{Results and Discussion}

By using 2DE coupled with MALDI-TOF MS, we have established an extended proteome reference map of $B$. pseudomallei. We picked approximately 300 protein spots identified by the PDQuest software. However, 282 protein spots were successfully identified by the PMF searching. PMFs of protein spots, which failed to be identified, showed only few peptide masses. In addition, those low-quality 


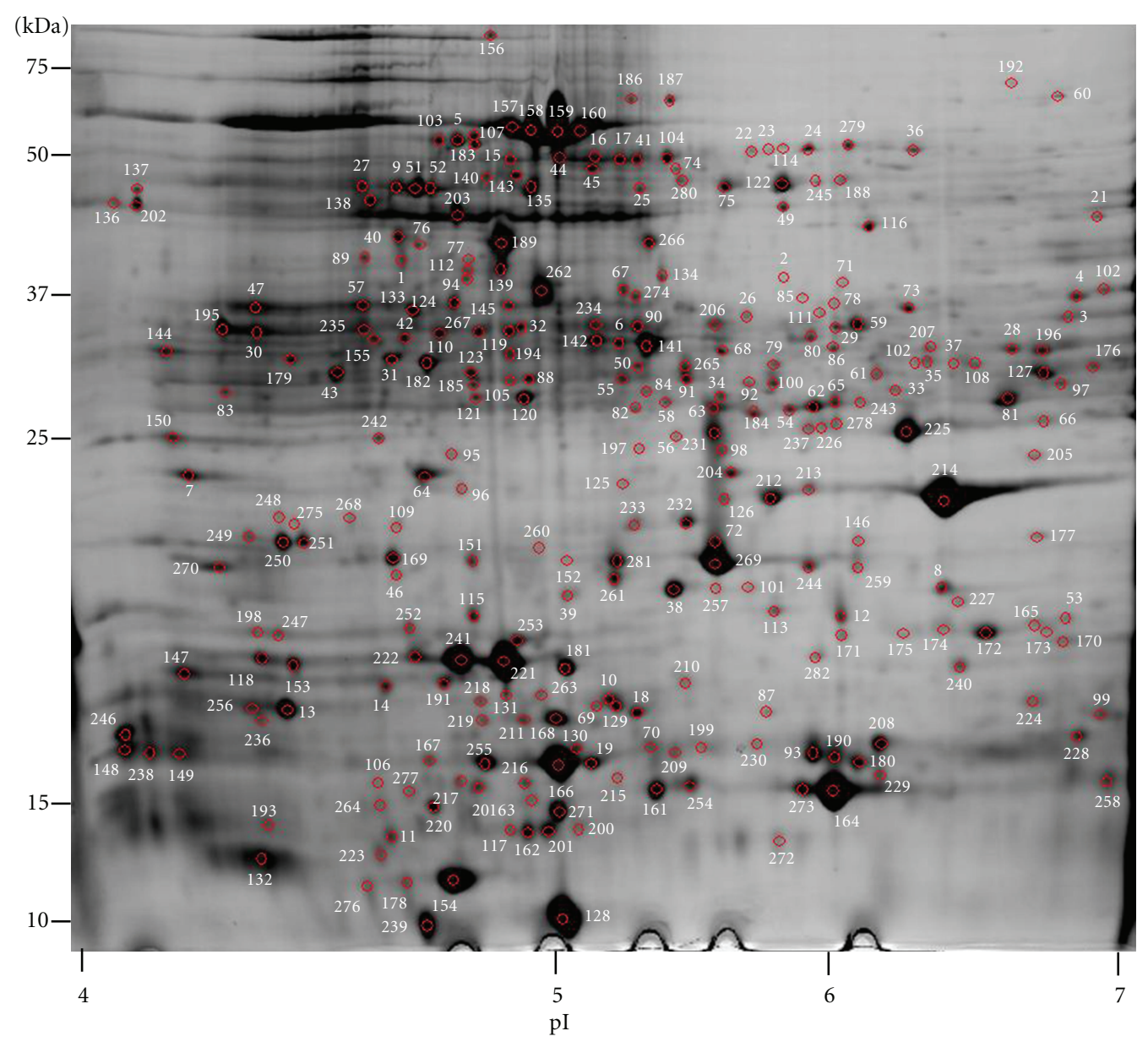

FIGURE 1: 2DE gel of B. pseudomallei proteome reference map grown under early stationary phase. The identified protein spots are listed in Table S1.

PMFs also resulted in matching with proteins from bacterial genus other than Burkholderia spp. rejecting the positive identification. The failure to identify those protein spots might be due to a very low protein amount of the excised spots. Figure 1 shows the identified 282 protein spots, representing 220 open reading frames, within the analysis window of $10-75 \mathrm{kDa}$, and pI 4-7. The identified protein spots were categorized according to their biological processes and were listed (see Table S1 in Supplementary material available online at doi: 10.1155/2011/530926). Based on a published genome of $B$. pseudomallei [4], we have thus compared our identified proteins with predicted ORFs from the genome. The result shows that proteins involved in posttranslational modification and lipid metabolism represent more than $50 \%$ of predicted ORFs. In contrast, only 1 and $2 \%$ have been identified as cell envelope biogenesis and outer membrane proteins and hypothetical proteins, respectively (Figure S1). Within our proteome reference map, over 97 spots equivalent to $35 \%$ of the identified ORFs are found to be involved in central metabolisms of the bacterium, of which energy, lipid, and carbohydrate metabolisms are the major pathways contributing to 28,18 , and $16 \%$, respectively (Figure S2). This result suggests an essential role of those gene products for survival of $B$. pseudomallei at this growth phase. Approximately 20\% of identified proteins presented in the reference map represent proteins functioning in posttranslational modification and stress responses. Within these categories, chaperonin GroEL, heat shock Hsp20-related protein, and phasin are highly expressed at both early and late stationary phase when compared with our previously partial B. pseudomallei proteome map [7]. Interestingly, up to $13 \%$ of the identified genes matched to hypothetical proteins highlighting the need for further functional characterization of these open reading frames, which might be important for the adaptation process upon entry into stationary phase.

We could also assign a new biological category of proteins identified in this extended proteome reference map, which we were unable to detect in the previous map. This group is genes associated with cell motility and intracellular trafficking and secretion and comprises $6 \%$ of the identified ORFs. Given the fact that the early stationary phase-associated adaptation involves expression of genes related to various processes of chemotaxis [11] and that expression of genes involved in cell trafficking and motility is down-regulated in bacterial prolonged culture [13], this might be the reason 
why we could observe this gene category at the early but not late stationary phase. Within this group, expression of genes involved in type III secretion systems BsaR, BsaU, BipB, and BipD (Table S1) has been reported to be induced by osmotic stress through a transcriptome analysis [14]. Similar to osmotic stress, a transcriptomic study has found that iron stress results in modulation of expression of ATP synthase subunit epsilon and succinate dehydrogenases [15], two proteins identified in our proteome reference map. These data show that our proteome reference map will be useful for studying comparative proteomics of $B$. pseudomallei grown under environmental stresses.

The B. pseudomallei genome consists of two chromosomes, in which the large chromosome (chromosome 1) contains genes necessary for central metabolism whereas the small chromosome (chromosome 2) harbors accessory genes important for bacterial response to the environment [4]. To elucidate which biological processes of the gene ontology classification are dominant to which chromosome, we grouped all identified genes according to their encoding chromosome (Figure S3). We show that expressed proteins belonging to nucleotide metabolism, coenzyme metabolism, translation, and posttranslational modification categories are dominant for chromosome 1. On the other hand, chromosome 2 favors proteins with secondary metabolism, cell motility, intracellular trafficking and secretion, and uncharacterized and hypothetical proteins. Since proteins are the functional output of genes, our result suggests a partitioning preference in functional expression of different cellular processes by the two chromosomes in B. pseudomallei. Because proteins involved in central metabolism represent the most abundant identified proteins of our proteome reference map, we then took the advantage of the Kyoto Encyclopedia of Genes and Genomes (KEGG) database (http://www.genome.jp/kegg/) to map metabolic pathways relating to these identified proteins. Pathways including glycolysis, Krebs cycle, oxidative phosphorylation, pentose phosphate pathway, and fatty acid metabolism are summarized in Figure S4.

Our extended proteome reference map also includes 34 genes whose expression is associated with protein isoforms (Table S2) in comparison with only 6 genes reported in our previous finding [7]. The most abundant protein isoforms are encoded by phasin, comprising 9 protein spots. This finding is correlated with our previous work, which shows phasin as the major protein isoforms expressed in the late stationary phase $B$. pseudomallei proteome. The abundance of phasin isoforms in the early and prolonged stationary phase cultures of $B$. pseudomallei suggests that the storage of polyhydroxy alkanoate, which is a form of carbon source associated with phasin, is a common feature between these growth phases. However, in contrast to the previous reported, we find the majority of protein isoforms falling into the category of central metabolism. For example, two enzyme isoforms encoded by glyceraldehyde 3-phosphate dehydrogenase (GapA) gene show a similar molecular weight, but their pIs are 4.47 and 6.70. This roughly 3-unit charge difference might be due to an intermediate transition, glyceraldehyde 3phosphate-bound form of the enzyme localized at the acidic
pI $[16,17]$. Thus, this result suggests that enzyme isoforms with charge difference might represent an intermediate of its catalytic step.

We have identified a number of genes, whose function has been reported to be implicated in bacterial pathogenesis, in the current proteome reference map of $B$. pseudomallei (Table S1). For instance, MurF is an enzyme required for bacterial cell wall synthesis and antibiotic resistance. Ppk2, a gene belonging to polyphosphate kinase family, functions in polyphosphate and ATP/GTP biogenesis and signaling during stress response. Similarly, adenylate kinase and adenylate cyclase are involved in ATP metabolism and signaling. Type III secretion-related proteins are required for invasion of pathogenic bacteria into host cells. Expression of many of these proteins has been shown to be controlled by stress response sigma factors RpoS and RpoE in B. pseudomallei $[8,9]$. Thus, translating our proteome reference map to other comparative proteome studies of $B$. pseudomallei grown at early stationary phase shall benefit the study of pathogenesis caused by $B$. pseudomallei, since expression of these pathogenesis-related proteins might be regulated by bacterial intrinsic factors and growth condition.

With the very similarity in nucleotide and in amino acid sequences between B. pseudomallei, B. mallei, and B. thailandensis genomes $[3,4]$, we propose that our proteome reference map constructed from $B$. pseudomallei can also be applied to the other two species and will be useful for comparing their proteome profiles. To provide an example, we previously reported a comparative proteomic analysis in $B$. pseudomallei and the nonvirulent sibling $B$. thailandensis and identified a number of potential virulent factors and biomarkers for differentiating these two bacterial species [7]. This proteome reference map of proteins expressed at early stationary phase in B. pseudomallei will facilitate further studies on "melioidomics," that is, the study of melioidosisrelated global profile expressions.

\section{Conclusion}

The proteome reference map containing 282 protein spots, which represent 220 protein encoding genes, has been established for the melioidosis pathogen B. pseudomallei. Many of the identified proteins have been implicated in virulence of pathogenic bacteria and have been shown to be controlled by the stationary phase sigma factor RpoS and a biofilm-associated sigma factor RpoE.

\section{Abbreviations}

2DE: Two-dimensional gel electrophoresis ORFs: Open reading frames.

\section{Acknowledgment}

This work was supported by the Junior Science Talent Project (JSTP) together with the National Science and Technology Development Agency (NSTDA) and the Thailand Research Fund (TRF) to Mr. P. Wongtrakoongate (JSTP-01-46-14R). 


\section{References}

[1] N. J. White, "Melioidosis," The Lancet, vol. 361, no. 9370, pp. 1715-1722, 2003.

[2] M. Wheelis, "First shots fired in biological warfare," Nature, vol. 395, no. 6699, p. 213, 1998.

[3] W. C. Nierman, D. DeShazer, H. S. Kim et al., "Structural flexibility in the Burkholderia mallei genome," Proceedings of the National Academy of Sciences of the United States of America, vol. 101, no. 39, pp. 14246-14251, 2004.

[4] M. T. G. Holden, R. W. Titball, S. J. Peacock et al., "Genomic plasticity of the causative agent of melioidosis, Burkholderia pseudomallei," Proceedings of the National Academy of Sciences of the United States of America, vol. 101, no. 39, pp. 1424014245, 2004.

[5] R. A. Moore, S. Reckseidler-Zenteno, H. Kim et al., "Contribution of gene loss to the pathogenic evolution of Burkholderia pseudomallei and Burkholderia mallei," Infection and Immunity, vol. 72, no. 7, pp. 4172-4187, 2004.

[6] C. Ong, C. H. Ooi, D. Wang et al., "Patterns of large-scale genomic variation in virulent and avirulent Burkholderia species," Genome Research, vol. 14, no. 11, pp. 2295-2307, 2004.

[7] P. Wongtrakoongate, N. Mongkoldhumrongkul, S. Chaijan, S. Kamchonwongpaisan, and S. Tungpradabkul, "Comparative proteomic profiles and the potential markers between Burkholderia pseudomallei and Burkholderia thailandensis," Molecular and Cellular Probes, vol. 21, no. 2, pp. 81-91, 2007.

[8] V. Thongboonkerd, M. Vanaporn, N. Songtawee et al., "Altered proteome in Burkholderia pseudomallei rpoE operon knockout mutant: insights into mechanisms of rpoE operon in stress tolerance, survival, and virulence," Journal of Proteome Research, vol. 6, no. 4, pp. 1334-1341, 2007.

[9] Y. Osiriphun, P. Wongtrakoongate, S. Sanongkiet, P. Suriyaphol, V. Thongboonkerd, and S. Tungpradabkul, "Identification and characterization of RpoS regulon and RpoS-dependent promoters in Burkholderia pseudomallei," Journal of Proteome Research, vol. 8, no. 6, pp. 3118-3131, 2009.

[10] P. Pumirat, P. Saetun, S. Sinchaikul, S. T. Chen, S. Korbsrisate, and V. Thongboonkerd, "Altered secretome of Burkholderia pseudomallei induced by salt stress," Biochimica et Biophysica Acta, vol. 1794, no. 6, pp. 898-904, 2009.

[11] M. Schuster, A. C. Hawkins, C. S. Harwood, and E. P. Greenberg, "The Pseudomonas aeruginosa RpoS regulon and its relationship to quorum sensing," Molecular Microbiology, vol. 51, no. 4, pp. 973-985, 2004.

[12] P. Utaisincharoen, N. Tangthawornchaikul, W. Kespichayawattana, P. Chaisuriya, and S. Sirisinha, "Burkholderia pseudomallei interferes with inducible nitric oxide synthase (iNOS) production: a possible mechanism of evading macrophage killing," Microbiology and Immunology, vol. 45, no. 4, pp. $307-$ 313, 2001.

[13] C. Pesavento, G. Becker, N. Sommerfeldt et al., "Inverse regulatory coordination of motility and curli-mediated adhesion in Escherichia coli," Genes and Development, vol. 22, no. 17, pp. 2434-2446, 2008.

[14] P. Pumirat, J. Cuccui, R. A. Stabler et al., "Global transcriptional profiling of Burkholderia pseudomallei under salt stress reveals differential effects on the Bsa type III secretion system," BMC Microbiology, vol. 10, article 171, 2010.

[15] A. Tuanyok, H. S. Kim, W. C. Nierman et al., "Genome-wide expression analysis of iron regulation in Burkholderia pseudomallei and Burkholderia mallei using DNA microarrays," FEMS Microbiology Letters, vol. 252, no. 2, pp. 327-335, 2005.
[16] M. T. Ryzlak and R. Pietruszko, "Heterogeneity of glyceraldehyde-3-phosphate dehydrogenase from human brain," Biochimica et Biophysica Acta, vol. 954, no. 3, pp. 309-324, 1988.

[17] J. M. Armstrong and D. R. Trentham, "The reactions of D glyceraldehyde 3 phosphate with thiols and the holoenzyme of D glyceraldehyde 3 phosphate dehydrogenase and of inorganic phosphate with the acyl holoenzyme," Biochemical Journal, vol. 159, no. 3, pp. 513-527, 1976. 

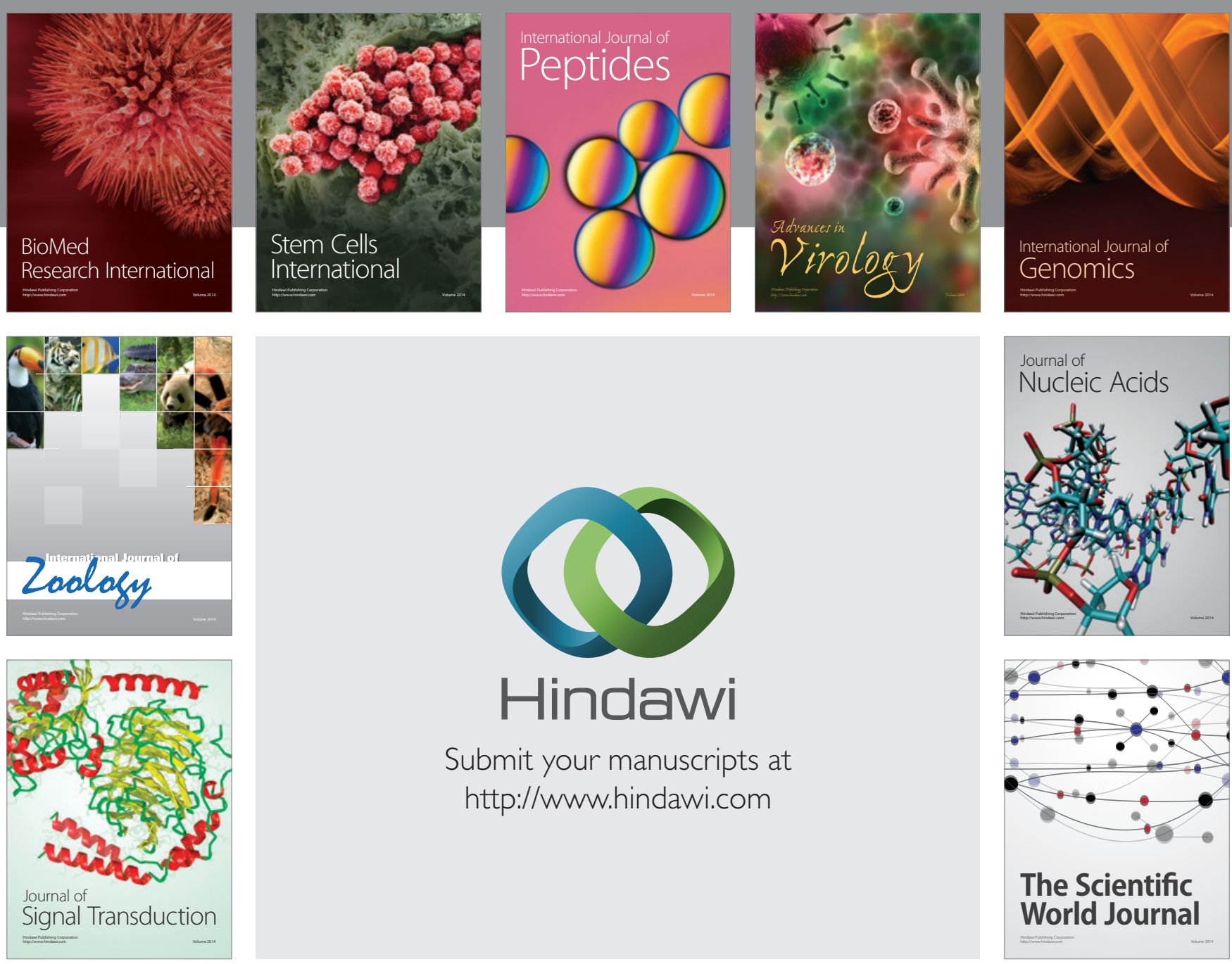

Submit your manuscripts at

http://www.hindawi.com
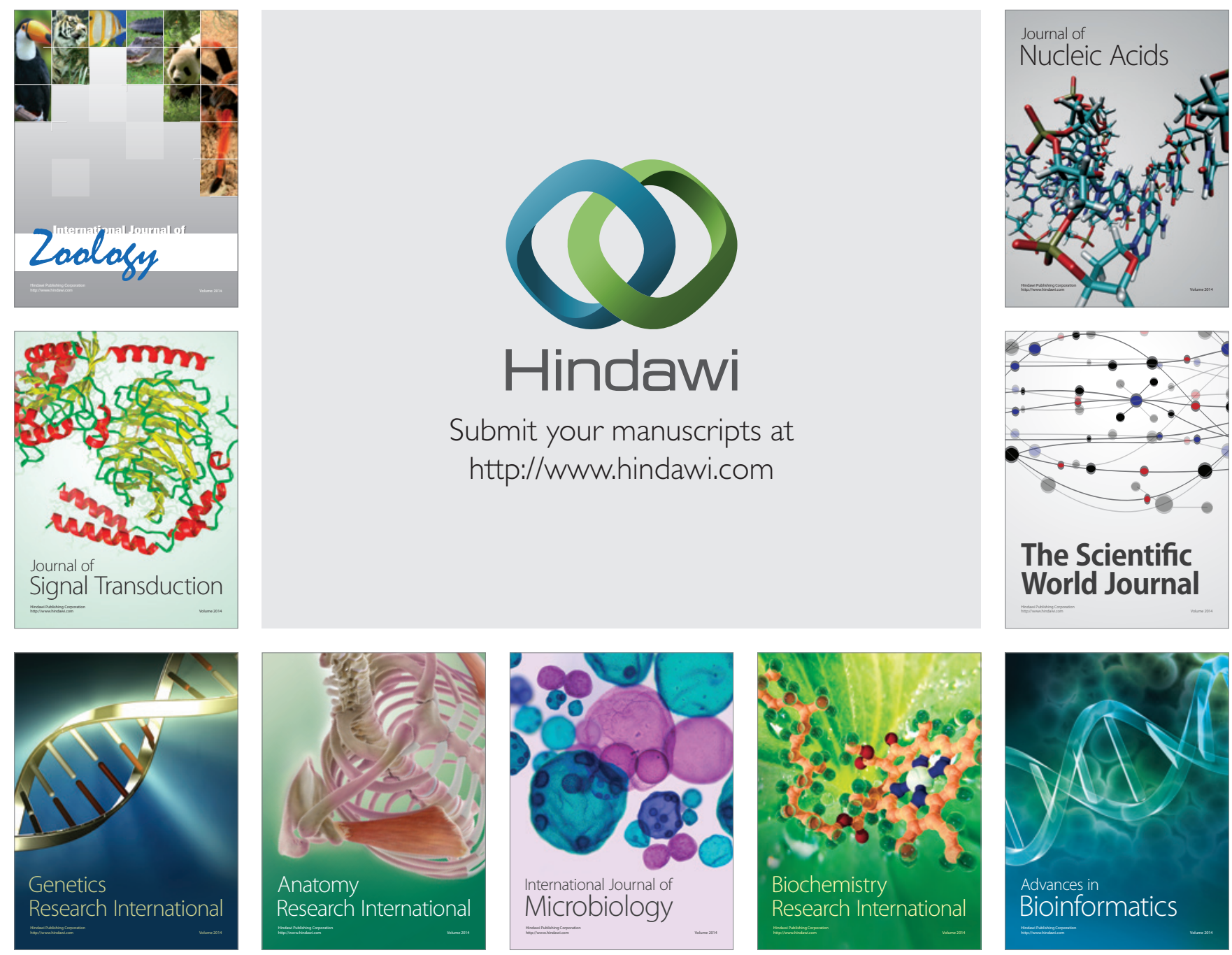

The Scientific World Journal
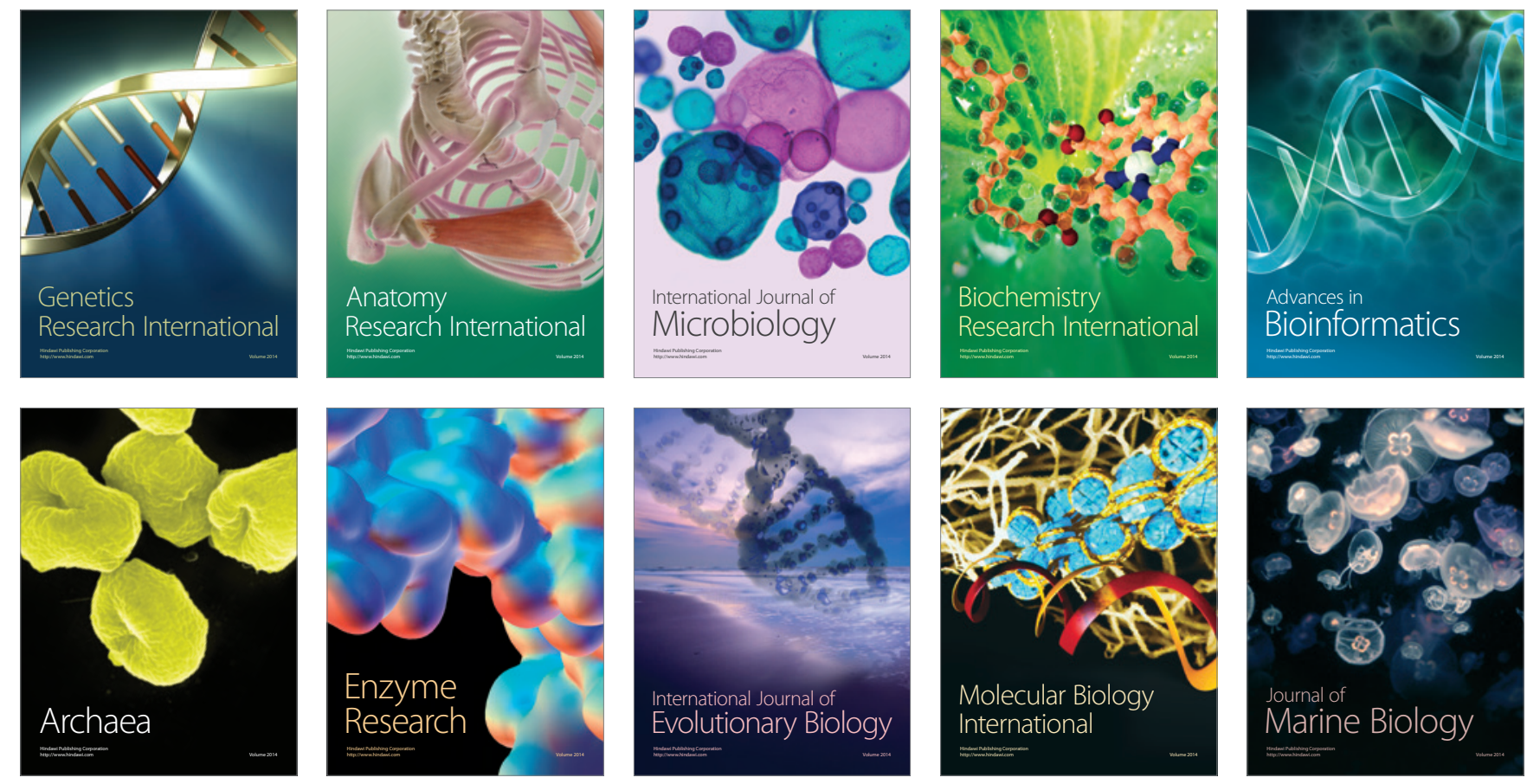